Construct Validity and Psychometric Properties of T-MoCA

Construct Validity and Psychometric Properties of the Tamil (India) Version of Montreal Cognitive Assessment (T-MoCA) in Elderly

Mani Abdul Karim ${ }^{12 *}$ (D) \& Jonnadula Venkatachalam ${ }^{3}$ (D)

1* Corresponding Author, Ph.D. Research Scholar, Department of Psychology, Periyar

University, Tamil Nadu, India prfi2012@gmail.com

${ }^{2}$ Research Trainer, 7d PhytoNeural Research Lab, Coimbatore, Tamil Nadu, India

${ }^{3}$ Professor, Department of Psychology, Periyar University, Salem - 636011, India

NOTE: This manuscript has been published and is available in International Journal of Gerontology, October 2022, Volume 16, Issue 4.

http://www.sgecm.org.tw/ijge/

doi: 10.6890/IJGE.202210_16(4).0010

Please cite this work as:

Karim MA, Venkatachalam J. Construct Validity and Psychometric Properties of the Tamil (India) Version of Montreal Cognitive Assessment (T-MoCA) in Elderly. Int J Gerontol. 2022;16(4):365-369.

\title{
APA Style:
}

Karim MA, Venkatachalam J (2022). Construct Validity and Psychometric Properties of the Tamil (India) Version of Montreal Cognitive Assessment (T-MoCA) in Elderly. Int J Gerontol. 16(4):365-369.

\section{For any queries/ comments/ suggestions:}

\section{Abdul Karim, Ph.D. Research Scholar,}

Department of Psychology,

Periyar University,

Periyar Palkalai Nagar,

Salem, Tamil Nadu - 636011

India

Phone: 919787337879

E-mail: prfi2012@gmail.com, karim@periyaruniversity.ac.in

Running Title: Construct Validity and Psychometric Properties of T-MoCA 
Construct Validity and Psychometric Properties of T-MoCA

\begin{abstract}
Background: The Montreal Cognitive Assessment (MoCA) is a neuropsychological cognitive tool developed and adapted widely in various languages for screening mild cognitive impairment (MCI).
\end{abstract}

Objectives: The present study aimed to evaluate the psychometric properties of the Tamil (India) Version of MoCA (T-MoCA) and further examine the construct validity of the tool.

Method: The authors conducted internal consistency, test-retest, sensitivity-specificity, and construct validity using 233 Tamil-speaking elderly participants. The inclusion criteria of the study participants were 0.5 or less than 0.5 scores in the Clinical Dementia Rating scale (CDR). Further, T-MoCA was used to screen MCI.

Results: The result showed that the T-MoCA had high internal consistency (0.83) and high test-retest reliability (0.92). Receiver operating characteristic (ROC) analyses showed an area under the curve (AUC) of 0.91 (95\% CI 0.87-0.94) for detecting MCI. Furthermore, the optimal cut-off score to detect MCI was 24 , accommodated a sensitivity and specificity of $88.4 \%$ and $77.9 \%$, respectively.

Conclusions: The Tamil (India) version of the MoCA maintained its core diagnostic properties, furnishing it a valid and reliable tool for the screening of MCI. Also, its latent dimensions help to understand the elders' cognitive function in a better way.

Keywords: T-MoCA, reliability and validity, cut-off score, factor analysis, elderly 